\title{
A Systems Thinking View on Cloud Computing and Energy Consumption
}

\author{
Michal Sedlacko / André Martinuzzi \\ Institute for Managing Sustainability \\ WU Vienna \\ Vienna, Austria \\ andre.martinuzzi@wu.ac.at
}

\author{
Karin Dobernig \\ Institute for the Environment and Regional Development \\ WU Vienna \\ Vienna, Austria \\ karin.dobernig@wu.ac.at
}

\begin{abstract}
Cloud Computing is often regarded as form of "green computing". However, the growth of cloud computing also goes hand in hand with an increasing demand for energy, caused by a continuously growing demand for content, data-intensive applications and bandwidth. The debates on the energy implications of the substantial shift to cloud computing among industry experts, researchers and civil society organizations mainly focus on stand-alone aspects of this shift, whereas a comprehensive view on the whole system, its inter-relations and driving forces are still rare.
\end{abstract}

In the course of an EU-funded project (www.SCPRESPONDER.eu) the syntax of causal loop diagrams and analytical tools of system dynamics has been used to develop a system map of the diverse effect pathways that a shift to cloud computing may have on energy consumption. The map was developed in an iterative participatory expert consultation process involving 25 experts from industry, research and policymaking. The map allows for integrating different strands of knowledge, shedding light on the dynamics of the whole system and identifying leverage points for business strategies as well as policy interventions. It shows that the effects on overall energy consumption depend on (1) relative strengths of the individual effect pathways which are, inter alia, (2) dependent on energy efficiency gains achieved through a broad diversity of measures in very different areas, while (3) the key drivers of the whole system are economy of scales (through reductions of service cost per unit) and (4) new user needs for data-intensive services. While some experts perceive these dynamics as typical rebound effects (increased efficiency is compensated by growth), others regard them as success strategies for individual businesses and the sector as a whole. Both groups share the view that service reliability, privacy and security of cloud services are the most important leverage points of the whole system. Furthermore, even if, through the growth of data-intensive services, the shift to cloud computing would cause growth of the overall energy consumption, this increase might potentially be off-set by thirdorder effects in the form of savings in the areas of mobility or housing (e.g. teleworking).

Index Terms: cloud computing, energy consumption, systems thinking, system dynamics

\section{INTRODUCTION}

Cloud Computing has emerged as an umbrella term to describe a category of on-demand computing services that were initially offered by commercial providers such as Amazon, Google and Microsoft. It presents a model on which a computing infrastructure is viewed as a "cloud" from which business and individuals can access applications from anywhere in the world upon demand; thus, cloud computing enables data to be delivered directly from the Internet to an electronic device rather than being stored on the device itself. The main principle behind this model is to offer computing, storage and software "as a service" [1].

Cloud Computing appears to be environmentally friendly due to the economies of scale created by cloud computing resources and facilities. On the individual consumer level, some cloud computing services replace offline activity that is equally or more energy intensive, and hence make a positive contribution to emissions reduction (e.g. digital purchase of music, online browsing of newspapers, Skype audio or video meetings, etc.). However, the growth of cloud computing is also accompanied by an increasing demand for energy. For all of this content to be delivered to users in real time, virtual mountains of video, pictures and other data must be available for almost instantaneous access and stored in massive data centres. These data centres are the fastest growing cause of IT energy use. Data centres currently consume $1.5 \%$ to $2 \%$ of global electricity - this figure is also expected to grow at a rate of $12 \%$ per year [2].

According to the CISCO VNI forecast, consumer traffic was responsible for around $80 \%$ of bandwidth use in 2009 , and is expected to grow at a faster rate than business traffic. One of the main drivers of this increased demand is the expansion of cloud computing. This means that content and services are increasingly offered online over a "cloud": video material uploaded and downloaded through YouTube, on-demand TV programs through apps such as the BBC iPlayer, books and maps through Google, news through newspaper and TV channel sites, music downloads through iTunes, audio and video connectivity through Skype, music streaming through Spotify, and so on. Moreover, the rise of social networking sites has encouraged uploading and sharing of material causing 
informal viral publicity to spread the information about interesting or amusing media quickly [3].

Hence, the net environmental impacts of cloud computing are still not clear. This is also due to a lack of utilization data from the major cloud companies. Much greater transparency is needed from data centre operators on their energy footprint for advancing the debate among peers and government regulators and substantiating the claims of 'green IT'.

Based on this rather diverse and scattered evidence our research aimed at the following:

- integrating different debate strands on cloud computing and energy consumption into a broader picture,

- considering systems dynamics (such as positive and negative feedback loops), and

- identifying leverage points for policy interventions and future research needs.

\section{THE RESPONDER METHOD}

The EU funded project RESPONDER (www.scpresponder.eu) aimed at developing and testing an innovative method for knowledge brokerage between research and policy making. In doing so, we follow the transactional network understanding of knowledge brokerage and the perspective of systems thinking. Building on participatory modelling approaches and applications (see [4], [5], [6], and [7]) we developed a method called 'participatory systems mapping' (PSM). Application of PSM can be best described as the preparation and implementation of a facilitated group process for developing and analysing causal loop diagrams that provide insight into a particular problematic issue and enable knowledge exchange.

Causal loop diagrams (CLDs) is probably the most-utilised systems-thinking visualisation tool since the 1960s (see Forrester, 1968). They have been specifically shaped by systems dynamics and cybernetics. Two widely recognised uses of CLDs are (1) the transformation of verbal descriptions into a feedback structure during early stages of model conceptualisation [8], and (2) the presentation of a 'distilled' understanding at the end of the whole modelling process [9]. Since an underlying principle of systems thinking is that the behaviour of a system is the result of the structure of its elements, a CLD provides an endogenous explanation for this observed behaviour.

In the RESPONDER project we utilized CLDs in the already established way while at the same time tested their use for knowledge brokerage. In more detail, we used CLDs to:

(i) transform perceptions and mental models of individuals and groups into causal and feedback structures,

(ii) expand thinking boundaries by enabling exploration and exchange of knowledge between paradigmatic and value positions accepted in various communities involved in the process, (iii) identify knowledge gaps by comparing them with evidence-based and systematised knowledge, and

(iv) formulate hypotheses about causes and effects, gather insights into system's behaviour and identify potential leverage points.

The RESPONDER project held 13 events and over 70 facilitated sessions in which the participants from both science and policy participated in developing CLDs. We focused on five different areas of consumption (food, mobility, housing, ICT and finance). In total five PSM sessions were devoted to developing a systems thinking view on cloud computing and energy consumption.

1. During the first stage experts from the project consortium identified 'problem issues', drafted a discussion input paper based on a literature review and prepared the following PSM sessions.

2. The second stage consisted of two approx. 90min PSM sessions held within a 2 day workshop. The purpose of these sessions was to explore the problem issue, reflect on its systemic structure, address controversies, and, in the process, exchange knowledge on the issue as well as on the actors, discourses and worldviews around it.

3. During the third stage project consortium experts 'cleaned up', processed and digitalized the CLDs, reflecting (to the highest degree possible) the interests and concerns of the participants expressed while developing the map. Processed CLDs were uploaded to the RESPONDER online knowledge brokerage platform in order to enable documentation of and further interactive engagement with the CLDs. In addition, we sent the CLDs to a few renowned experts in the respective issue area and carried out expert interviews in order to review, validate and/or amend the CLDs. By processing the CLDs and documenting the workshop sessions, this stage also provided a basis for identifying and framing the possible foci for the next stage.

4. The fourth stage consisted again of two approx. 90min. PSM sessions held within a 2 day workshop. While the first participatory stage was more 'diagnostic', the second stage was more 'envisioning' and oriented towards problem solving by using the CLDs developed earlier as a starting point. Again, we also followed the objective of knowledge exchange.

5. The fifth stage consisted of updating the CLDs based on the workshop results and collecting and documenting participant inputs.

6. During the sixth stage a number of thematic publications were developed (which this paper is one of). In doing so the references to most recent publications were added and reviewers' comments were considered. 
Compared to other methods for structuring issue areas and knowledge (such as mind maps, cross-tables or scenarios) CLDs offer a rather simple standardized but still powerful 'language' consisting of four key elements:

1. Factors help explain a particular problem, provide a shared understanding of the components of a system among the participants and link CLD to existing knowledge. They should be measurable in scale and units.

2. Causal links represent positive or negative causal relationships between factors. Positive relationships indicate that factors develop in the same direction (an increase in $\mathrm{X}$ will lead to an increase in $\mathrm{Y}$ (assuming all other variables remain constant) and vice versa, a decrease in $\mathrm{X}$ will lead to a decrease in $\mathrm{Y}$. A negative causal relationship (marked in dotted lines in the following graph) is inverse; $\mathrm{X}$ and $\mathrm{Y}$ move in opposite directions.

3. Feedback loops are circular causalities that provide the system with a continuous dynamic. Positive (also called reinforcing) feedback loops are often responsible for exponential growth or exponential decay as the system sustains and even reinforces the initial move. Since unlimited exponential growth is impossible in closed systems, it is often useful to search for the limits to growth. Negative (also called balancing) feedback loops tend to have the self-regulating and stabilizing effects on systems as the system fights against the initial move (in case of time delays these balancing feedback loops can create oscillations).

4. Colors were used to identify different causal pathways in order to make complex system maps easier to understand, and tell different stories within one map or even represent different world views in one map.

\section{THE RESPONDER CLOUD COMPUTING MAP}

The RESPONDER Cloud Computing Map (see below) presents a systemic view on the dynamic interrelations between the adoption of cloud computing services and energy consumption. It depicts integrated mental models of policy, research and industry representatives who participated in our mapping sessions. A systemic perspective on the complex relations between cloud computing and energy consumption allows for the map to bring together the supply and demand side of cloud computing services, as well as consider different levels and units of analysis (e.g. data centers, networks, individual users, and individual user devices). In other words, the map helps to capture co-dynamics on the macro, meso and micro levels. Integrating these perspectives into one map provides an important contribution to the current debate, and potentially helps identify existing research gaps and leverage points for policy intervention.

In the following sections, we present the developed system map through five pathways that are displayed in different colors in the map (see previous section). We thereby move from the macro-level perspective of data centers (supply side) over the meso-level (networks) to the micro-level (individual users and devices). All five pathways depict the systemic relationship between the usage of cloud services and energy consumption. The relative 'size' or 'strength' of the individual pathways indicate whether, or in how far, the adoption of cloud services can lead to savings in energy consumption.

\section{Dynamic I: Centralization vs. Decentralization of Computing}

The first perspective deals with the replacement of in-house data centers with shared facilities (i.e. external, big data centers operated by cloud service providers) and thus a centralization of computing activity. As was already mentioned in the introductory section, the data needs of ongoing adoption of cloud services are increasingly handled by external data centres. Thus, the energy consumed in the system shifts from in-house computing (see [10], [11], and [12]) to energy consumption in data centres (see [13], [14], [15], [16], [17], and [18]). In the map, this means an increase in 'size' of the black pathway and a decrease in 'size' of the red pathway. This development is reinforced through economies of scale (which is depicted as a reinforcing feedback loop in the map). The more data centres are build or the higher their capacity, the cheaper one unit of service becomes. These falling unit costs encourage companies to outsource even more of their computing needs to data centres. Savings mainly result from a more efficient use of hardware as well as a reduced need for support teams [19].

The decisive factors when it comes to overall energy consumption in this dynamic are (1) the level of energyefficiency of in-house data centers versus big data centers, and (2) the type or quality of energy consumed through the respective data centers. Concentrating computing into several big data centres in a given geographical location might allow agreeing on a specific type of energy with the energy provider. This bares a high potential to increase the usage of renewable energy compared to decentralized decisions by a high number of in-house data centres. However, while many considerations go into determining where new data centres could be located - looking at reliable and low-cost source of electricity, tax incentives and proximity to end-user - the availability of renewable energy to power the data centre is currently low on most cloud companies' lists of consideration [20]. Instead, data centre clusters are cropping up in places where cheap and dirty coal-powered electricity is abundant. The greatest effort to reduce the environmental footprint of data centres has so far concentrated on efficiency gains through e.g. improving data centre design, increasing server energy efficiency or reducing waste associated with cooling and other 'non- computing' energy demands. Putting more focus on the kind of energy used to feed present consumption is an important leverage point for possible policy interventions. 


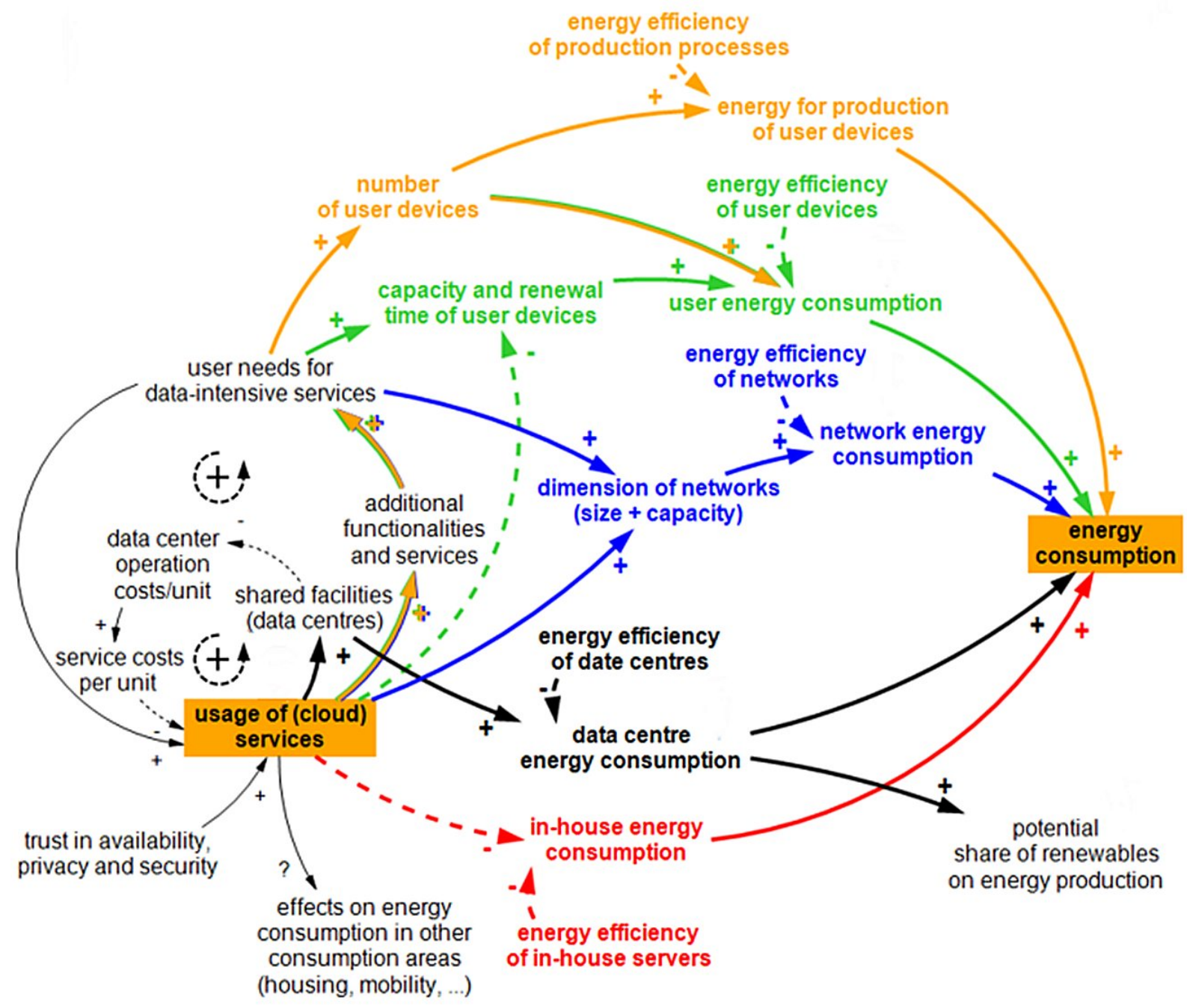

Fig. 1. Cloud Computing System Map 


\section{Dynamic II: Co-Evolution of Supply and Demand of Cloud Services}

The second dynamic that has been captured through the participatory system mapping process relates to the coevolution of supply of and demand for cloud computing services. Cloud computing services are meant to replace offline activities that are energy and resource intensive. However, cloud services are also accompanied by new functionalities and services that in turn create additional user needs. When these functionalities and services are desired and expected by consumers, they increase the need for data-intensive services. The more normalised they become, the more they contribute to further growth of cloud services. In addition, they potentially induce consumer network traffic to intensify, also because ICTs allow more resource consumption per unit of time, for example through activation of dead time and multi-tasking possibilities. This demand-pull effect constitutes another reinforcing feedback loop and presents a potential rebound effect - new functionalities create new user needs which cause even more network traffic than would otherwise be required for the previously existing computing needs (see [21], [22], and [23]). In the map, the blue pathway depicts the increase of network traffic and the resulting energy consumption through networks as a result of a heightened collective demand for cloud computing services (see [24] and [25]).

\section{Dynamic III: Efficiency, Substitution and Accumulation of User Devices}

The third perspective displayed in the system map deals with the level of individual user devices. In the map, the green pathway captures the effects of user devices on energy consumption in the usage phase. With an increase in cloud computing, devices can become simpler (i.e. have less internal computing capacity) and can also last longer. An increase of cloud computing therefore would result in lower energy consumption by the user's device than it would have been otherwise. A key promise of cloud computing is the dematerialization of physical products, services and processes which could result in vast reductions in energy and materials consumption with consequent reductions in greenhouse gas emissions (see [26] and [27]). Consumption of digital goods in many areas can help reduce $19 \%$ of global GHG emissions produced by manufacturing industries (see [28], [29], and [30]). However, the rebound effect outlined in the previous section can also potentially lead to increaisng the number of user devices. With new functionalities and services made available by cloud computing, the capacity requirements for user devices (and therefore their energy consumption) will grow [31]. The decisive issue is thus whether cloud computing essentially leads to a substitution or accumulation of user devices.

Furthermore, user devices do not only consume energy in the usage phase but also in the production phase. The orange pathway in the map captures the energy required for the production of user devices. As previously mentioned, the spread and normalisation of functionalities and services enabled by growth of cloud computing might result in increasing consumer demand for cloud-compatible devices [32]. Naturally, the more devices are in use, the higher would be the total user energy consumption caused by their production.

Overall, the critical factor in explaining and calculating the effects of cloud computing on energy consumption is the energy efficiencies of the outlined, individual pathways. Efficiencies regulate the overall energy flows 'through' these pathways. Furthermore, a number of contingent thirdorder effects are also depicted in the map. For example, an increased adoption of cloud services could have effects on the way people work - say, it could enable a rise in teleworking which has impacts on household energy consumption through heating as well as on energy consumption through transportation. It could also change how people shop (online shopping for single items, each delivered separately) and cause changes in many other areas (see [33] and [34]). Yet, the enabling and limiting factor for usage growth of cloud services is the trust in service reliability, trust in privacy of data, and trust in security (see [35] and [36]).

\section{EXPERIENCES AND LIMITATIONS}

Bellow we present our key identified experiences in applying the PSM method while developing the cloud computing map: A) Preparation, B) Scoping and Framing, C) Facilitation, D) Timing and Motivation, E) Knowledge Co-Production and F) Bridging World-Views.

A) Preparation: Preparation required identifying and formulating the problem issues to be explored beforehand. For practical purposes we decided to define problem issues through expert input, rather than participatory input. Drawing on the systems thinking competence of the consortium, we made sure that the problem issues were viable for mapping, and desirably depicted unexpected or counterintuitive developments that could lead to useful insights. This involved formulating the issues at an appropriate level of abstraction and complexity.

B) Scoping and Framing: PSM is easier when it deals with a concrete system all participants are familiar with (e.g. a corporate or local problem) and gets rather difficult when more abstract terms (such as competitiveness or responsibility) are used. Therefore, scoping and framing of each PSM workshop are key. In addition, CLDs can become rather large and complex if the scope of the assessed system is unclear and participants bring in more and more aspects rather than focusing on the key components and dynamics. Instead of starting lengthy debates about the scope of the system we used two factors as starting points implicitly framing the system (in our case 'usage of cloud services' and 'energy consumption') as well as a guiding question ('which are the systemic links of the usage of cloud services and the total energy consumption?'). Both helped the facilitators to sort out inputs (e.g. by assessing 
whether the newly introduced element in the map helped answer the guiding question), avoid overcrowding the system and (re-)focus the debate on the key question of the PSM workshop.

C) Facilitation: In our experience the skills and training of facilitators should not be under-estimated. The PSM method requires a facilitator to possess three kinds of expertise simultaneously: systems dynamics/cybernetics expertise, facilitation and moderation skills, and background knowledge related to the issue being mapped. The facilitation as such is very different to many other participatory methods as it requires to slow down the inputs (in order to ensure that all participants can follow), match inputs to what is already in the map (in order to avoid clustering instead of system mapping) and to park or even reject input (in case it is not related to the question in focus). During the RESPONDER project we carried out a series of trainings, role-plays and trials before starting with real-life PSM moderation. It also proved helpful to support the facilitator with a rapporteur who took notes and kept the unprocessed input ad notam.

D) Timing and Motivation: The viable length of a single PM session seems to lie between 90 and 120 minutes as it takes a certain time to enter a productive 'mapping mode' and longer sessions tend to end up with too complex CLDs and run the risk of frustrating participants ('it looks like spaghetti'). In our experience the motivation of participants is higher, if a second round of PSM (after a longer break or on a second day) builds on the output of the first round and sufficient time is taken for reflecting on the process and the results. It can support the PSM process if the output of the first session is processed before the second session starts (e.g. by re-arranging factors and loops), however, continuity and ease of understanding must be ensured in order to avoid confusion.

E) Knowledge Co-Production: In the first round of PSM sessions the mapping was exploratory and diagnostic as the participants collaboratively created CLDs, discussed system elements, linkages and dynamics and identified knowledge gaps. The most productive seem to be channeling participant inputs through the facilitator into the map and for the facilitator to focus the attention of all participants on the issue discussed. In the second round of PSM sessions we used a range of exercises to support further insights on the basis of the maps produced earlier. These included (i) inferring system behaviour and identifying leverage points; (ii) translating policy options into the CLDs and tracing their potential expected and unexpected effects; and identifying knowledge gaps in order to formulate a research agenda. While the participants of the first round of PSM sessions evaluated the spirit of creativity and ownership extraordinarily highly, the satisfaction of the participants of the second round was substantially lower. Many of them would have liked to expand, re-structure, or expand the scope of the system maps (knowledge co-production) rather than applying them (knowledge brokerage).

F) Bridging World-Views: Compared to other issue areas the PSM sessions on cloud computing were less adversarial and the participants shared a mission of getting a glimpse on the bigger picture. On the other hand the participants of housing and mobility PSMs experienced conflicting world-views (e.g. green growth and eco-efficiency versus de-growth and sufficiency). Within these more contested areas PSM and CLDs turned out to provide a language that supported taking a broader perspective on the whole system, improving the understanding of world-views of other participants and going beyond individual disciplines.

\section{LIMITATIONS}

The influence of the facilitator and the expert group composition on the focus and structure of a CLD could be perceived as one of the most important factors limiting the validity of a CLD. In order to deal with this issue we carried out several PSM sessions with different groups in the RESPONDER project and compared the results. These comparative cases showed a rather high similarity of CLDs and therefore indicate a high validity of the results. In addition, we included several feedback phases with different high-level experts who commented on the draft versions of the maps. In doing so we experienced oscillations between asking for 'more details' and 'more focus'. Future research could carry out more formalized experiments on whether CLDs produced by different groups and facilitated by different moderators show a high degree of similarity and why so (e.g. by video recording and analysis).

Another bias might be introduced through the scoping and framing of the PSM sessions. One could argue that even the discussion input paper suggested a framing which could be different if a different group of people would be involved in this phase. In addition, our decision to define the issues beforehand clearly shifted the ownership of the process towards the project consortium. We did not entirely manage to prevent some of the participants feeling that there is a 'right' answer and that the facilitators are manipulating the process towards a predetermined system representation. In the preparation of the second round of sessions we therefore identified the focal points of the PSM sessions in a participatory manner (i.e. by short telephone interviews with a smaller proportion of the confirmed participants and additional experts in the field). Future research could experiment with more or less participatory methods for framing the PSM sessions and their influence on the produced CLDs.

System mapping aims to make participants' mental models explicit. However, people are not always aware of their mental models and are not always able to articulate them. Thus, one needs to differentiate between the output (the map) and the mental models of the participants. While the final maps run the risk of representing a smoothed surface, the mental models might stay diverse, ambiguous and implicit. Therefore, we suggest future research on embedding PSM in larger dialogue processes as well as the application of qualitative research methods to assess the effects of the PSM method on individual 
metal models and world views as well as on communities in involved.

\section{DISCUSSION AND OUTLOOK}

The RESPONDER cloud computing map shows that the effects of cloud computing on overall energy consumption depend on (1) relative strengths of the individual effect pathways, which are, inter alia, (2) dependent on energy efficiency gains achieved through a broad diversity of measures in very different areas, while (3) the key drivers of the whole system are economy of scales (through reduces service cost per unit) and (4) new user needs for data-intensive services.

While some experts perceive these dynamics as typical rebound effects (increased efficiency is compensated by growth), others perceive them as success strategies for individual businesses and the sector as a whole. Both groups share the view that service reliability, privacy and security of cloud services are the most important leverage points of the whole system. Furthermore, even if, through the growth of data-intensive services, the shift to cloud computing would cause growth of the overall energy consumption, this increase might potentially be off-set by third-order effects in the form of savings in the areas of mobility or housing (e.g. teleworking).

While there is a risk that new demands will overcompensate the reductions of total energy consumption, policy interventions can be made easier when decision power is concentrated in the hands of a small number of companies (e.g. in order to increase the use of renewable energy). While discussing our findings with experts from the ICT sector it became obvious that by now no such leverage points for policy interventions have been identified. This could be a promising area of future research.

\section{ACKNOWLEDGMENT}

Research presented in this paper has been conducted as part of the RESPONDER project funded by the Seventh EU Framework Program for Research and Technological Development (FP7; grant agreement no. 265297). The authors would like to thank Nuno Videira and Paula Antunes from the Center for Environmental and Sustainability Research at Universidade Nova de Lisboa and Inge Røpke from the Center for Design, Innovation and Sustainable Development at Aalborg University for the valuable input in developing and implementing the participatory system mapping method in the area of cloud computing, as well as all the experts who participated in the RESPONDER events.

\section{REFERENCES}

[1] Voorsluys, W., Broberg, J., and Buyya, R. (2011), Introduction to Cloud Computing, in R. Buyya, J. Broberg, and A. Goscinski, (Eds.): Cloud Computing: Principles and Paradigms, John Wiley \& Sons, Inc.
[2] Greenpeace. (2011). How dirty is your data? A Look at the Energy Choices That Power Cloud Computing: Greenpeace International.

[3] Preist, C., and Shabajee, P. (2010). Energy Use in the Media Cloud: Behaviour Change, or Technofix? Paper presented at the 2nd IEEE International Conference on Cloud Computing Technology and Science.

[4] Richardson, G. and Anderson, D. (1995), 'Teamwork in group model-building’, System Dynamics Review, 11 (2), 131-137.

[5] Vennix, J. (1996), Group Model-Building: Facilitating Team Learning Using System Dynamics, John Wiley \& Sons: Chichester.

[6] van den Belt, M. (2004), Mediated Modeling: A System Dynamics Approach to Environmental Consensus Building, Island Press: Washington DC.

[7] Videira, N., P. Antunes and R. Santos (2009), 'Scoping river basin management issues with participatory modelling: the Baixo Guadiana experience', Ecological Economics, 68 (4), 965-78.

[8] Goodman, M.R. (1974), Study Notes in System Dynamics, Cambridge, MA: MIT.

[9] Morecroft, J.D.W. (1982) 'A critical review of diagramming tools for conceptualising feedback system models', Dynamica, 8, 20-29.

[10] Accenture. (2010). Cloud Computing and Sustainability: The Environmental Benefits of Moving to the Cloud.

[11] Borderstep Institut für Innovation und Nachhaltigkeit. (2012). Gutachten zum Thema „Green IT - Nachhaltigkeit“ für die Enquete-Kommission Internet und digitale Gesellschaft des Deutschen Bundestages. Berlin.

[12] BITKOM (2008), Cloud Computing - Was Entscheider Wissen Müssen, Ein ganzheitlicher Blick über die Technik hinaus Positionierung, Vertragsrecht, Datenschutz, Informationssicherheit, Compliance http://www.bitkom.org/files/documents/BITKOM_Leitfaden_Cl oud_Computing-Was_Entscheider_wissen_muessen.pdf

[13] The Climate Group. (2008). SMART 2020. Enabling the low carbon economy in the information age: The Climate Group and the Global eSustainability Initiative (GeSI).

[14] Bianchini, R., and Rajamony, R. (2004). Power and energy management for server systems. Computer, 37(11), 68-74.

[15] Koomey, J. (2007). Estimating Total Power Consumption by Servers in the U.S. and the World. Oakland, CA.

[16] US EPA. (2008). Report to Congress on Server and Data Center Energy Efficiency Public Law 109-431 U.S. Environmental Protection Acency.

[17] IZM/ISI, F. (2009). Abschätzung des Energiebedarfs der weiteren Entwicklung der Informationsgesellschaft Abschlussbericht an das Bundesministerium für Wirtschaft und Technologie. Berlin, Karlsruhe.

[18] Greenpeace. (2010). Make IT Green. Cloud Computing and its Contribution to Climate Change: Greenpeace International.

[19] Verdantix. (2011). Cloud Computing - The IT Solution for the 21st Century Carbon Disclosure Project Study 2011: Carbon Disclosure Project.

[20] Greenpeace. (2011). How dirty is your data? A Look at the Energy Choices That Power Cloud Computing: Greenpeace International. 
[21] CISCO. (2014). CISCO Visual Networking Index: Forecast and Methodology 2012-2017. Retrieved March 5, 2014, from http://www.cisco.com/c/en/us/solutions/collateral/serviceprovider/ip-ngn-ip-next-generation-network/white_paper_c11481360.html.

[22] The Climate Group. (2008). SMART 2020. Enabling the low carbon economy in the information age: The Climate Group and the Global eSustainability Initiative (GeSI).

[23] Preist, C., Shabajee, P. (2010). Energy Use in the Media Cloud. Cloud Computing Technology and Scicnce, 2010 IEEE Second International Conference on Cloud Computing Technology and Science.

[24] Borderstep Institut für Innovation und Nachhaltigkeit. (2012). Gutachten zum Thema „Green IT - Nachhaltigkeit“ für die Enquete-Kommission Internet und digitale Gesellschaft des Deutschen Bundestages. Berlin.

[25] Baliga, J., Ayre, R., Hinton, K., and Tucker, R.S. (2010). Green Cloud computing: Balancing energy in processing, storage and transports. Proceedings of the IEEE, 99(1), 149-167.

[26] Mallon, K., G. Johnston, D. Burton, and J. Kavanagh. (2008). Towards a high-bandwidth, low carbon future: Telecommunications-based opportunities to reduce greenhouse gas emissions. Climate Risk Pty. Ltd.

[27] Pamlin, D., and K. Szomolanyi. (2008). "Saving the climate @ the speed of light. A report prepared for the European Telecommunications Network Operators Association (ETNO) and the World Wide Fund for Nature (WWF)".

[28] Weber, Christopher L., Jonathan G. Koomey, and H. Scott Matthews. (2010). The Energy and Climate Change Implications of Different Music Delivery Methods. Journal of Industrial Ecology no. 14 (5):754-769.

[29] Moberg, Å., and et al. 2010. Printed and Tablet e-Paper Newspaper from an Environmental Perspective - A Screening Life Cycle Assessment. Environmental Impact Assessment Review no. 30 (3):177-191.

[30] Kamburow, C. (2004). E-Paper-Erste Abschätzung der Umweltauswirkungen. Eine ökobilanzielle Betrachtung am Beispiel des Nachrichtenmediums Zeitung. Berlin: Institut für Zukunftsstudien und Technologiebewertung (IZT).

[31] Preist, C., Shabajee, P. (2010). Energy Use in the Media Cloud. Cloud Computing Technology and Scicnce, 2010 IEEE Second International Conference on Cloud Computing Technology and Science.

[32] Preist, C., Shabajee, P. (2010). Energy Use in the Media Cloud. Cloud Computing Technology and Scicnce, 2010 IEEE Second International Conference on Cloud Computing Technology and Science.

[33] Edwards, J. B., McKinnon, A. C., and Cullinane, S. L. (2010). Comparative analysis of the carbon footprints of conventional and online retailing: A "last mile" perspective. Int. J. Physical Distribution \& Logistics Management, 40(1-2), 103-123.

[34] Casal, C. R., Wunnik, C. V., Sancho, L. D., Burgelman, J. C., and Desruelle, P. (2005). How will ICTs affect our environment in 2020. Foresight, 7(1), 77-87.

[35] Verdantix. (2011). Cloud Computing - The IT Solution for the 21st Century Carbon Disclosure Project Study 2011: Carbon Disclosure Project.

[36] SIT. (2012). On the Security of Cloud Storage Services: Fraunhofer Institute for Secure Information Technology (SIT). 